\title{
Educación Mexicana Y Derechos Humanos
}

Lourdes Lavaniegos González *

\section{RESUMEN}

Uno de los derechos fundamentales de la humanidad y especialmente de los niños es el acceso a la educación; la ineficiencia de la educación mexicana que se ha dejado ver en los últimos años a nivel internacional, muestra que no se cumple con dichos derechos en nuestro país.

\section{ABSTRACT}

One of the main rights of humanity and specially of children is education, the inefficiency of the Mexican education, demonstrated through recently international investigations, proves that this main right is not been fulfilled.

\section{* Lourdes Lavaniegos González es Licenciada en Pedagogía por la Universidad Panamericana, Maestra en Educación por la Universidad Abierta y docente de la Universidad La Salle Pachuca en el área de Humanidades. INTRODUCCIÓN}

Uno de los primeros derechos de la humanidad es la posibilidad de educarse, tanto los organismos internacionales como la legislación de los diversos países del mundo lo dan por sentado; pero hoy día se habla de las carencias que sufre el docente, de la necesidad de dignificarlo y de los bajos resultados que arroja la práctica educativa, tanto en nuestra nación como en otros países.

En las siguientes páginas se analizan tanto los documentos internacionales acerca de los derechos humanos como la legislación mexicana en referencia a sus planteamientos educativos.

Los bajos resultados arrojados en los últimos años develan un foco de alarma en cuanto a la calidad de la educación mexicana; pero no sólo implican mala calidad, sino una ruptura de los derechos básicos y un incumplimiento de la normatividad constitucional y de la Ley General de Educación.

Es posible proponer soluciones, pero si no se llega al fondo del problema será difícil corregir el rumbo. A partir de la experiencia de la educación lasallista del siglo XVII parece existir una respuesta adaptable a las condiciones actuales del magisterio mexicano.

\section{Derechos Humanos y Educación}

A mediados del siglo XX, la Organización de la Naciones Unidas, preocupada por las frecuentes desigualdades y discriminaciones entre los seres humanos, emitió la Declaración Universal de los Derechos Humanos.

La mayoría de los países estuvieron de acuerdo con ellos; después de cincuenta años, en algunas regiones del mundo, siguen siendo el "deber ser" al que se quiere llegar. 
En el ámbito de la educación, el artículo $26^{\circ}$ dice:

1. Toda persona tiene derecho a la educación. La educación debe ser gratuita, al menos en lo concerniente a la instrucción elemental y fundamental. La instrucción elemental será obligatoria. La instrucción técnica y profesional habrá de ser generalizada; el acceso a los estudios superiores será igual para todos, en función de los méritos respectivos.

2. La educación tendrá por objeto el pleno desarrollo de la personalidad humana y el fortalecimiento del respeto a los derechos humanos y a las libertades fundamentales; favorecerá la comprensión, la tolerancia y la amistad entre todas las naciones y todos los grupos étnicos o religiosos, y promoverá el desarrollo de las actividades de las Naciones Unidas para el mantenimiento de la paz.

3. Los padres tendrán derecho preferente a escoger el tipo de educación que habrá de darse a sus hijos.

Existen, varias notas en la declaración que no se cumplen cabalmente en nuestro país:

En cuanto a la cobertura en la educación primaria apenas ha llegado, a principios de este siglo, a estar cerca del $100 \%$; pero el abandono es todavía alto y casi siempre está aparejado con problemas de índole económico. Para el año 2000, un $37 \%$ de los jóvenes habían dejado de estudiar antes de cumplir 15 años, y del $45 \%$ que habían dejado de estudiar entre los 15 y los 19 años el $67 \%$ quería seguir haciéndolo (Encuesta Nacional de la Juventud, 2000). Por tanto en la realidad, la cobertura tiene un alcance mucho menor que el que se afirma en las estadísticas y mientras no tengan todos los niños posibilidades de asistir a la educación básica, no se puede hablar de un derecho cubierto.

Un aspecto de menor importancia es que aunque la escuela básica se propone como "gratuita", existen diversas cuotas económicas, en especie, o en trabajo que las familias mexicanas deben aportar a los centros educativos. Esto no sería grave si pudiera estipularse con claridad, pero al no hacerse, la solicitud de cuotas queda al arbitrio de las autoridades escolares y asociaciones de padres de familia y corre el riesgo de observarse como ilegal. Exagerando un poco, podemos decir que la escuela no es del todo gratuita.

De mayor preocupación resulta lo mencionado en el segundo punto en cuanto a que la educación tendrá por objeto el pleno desarrollo de la personalidad humana, y que está lejos de ocurrir, si consideramos las carencias en la educación artística, física y espiritual de la escuela común. Un ejemplo claro es el tiempo dedicado a la educación física que en la escuela secundaria corresponde a una hora semanal, justamente en la edad en que el adolescente debe tener un amplio conocimiento de su cuerpo para llegar a la plenitud, se limita la práctica de manera alarmante. En peor estado se encuentra la educación artística que difícilmente cuenta con personal capacitado o la espiritual que ni siquiera se menciona en los planes de 
estudio oficiales. No puede existir pleno desarrollo humano si la intención educativa no lo prevé desde sus planes de estudio y desde la capacitación de sus docentes.

Tampoco se cumple la posibilidad de que los padres puedan elegir el tipo de educación que darán a sus hijos, al menos quienes no tienen los recursos para pagar una educación privada, dependen totalmente de las condiciones que las autoridades delimitan para la educación formal.

Por último, el acceso a los estudios superiores ni es igual para todos, ni depende de los méritos respectivos, ya que la cobertura universitaria pública es insuficiente y la universidad privada no tiene subsidio alguno que permita el acceso abierto a sus servicios como ocurre en otros países.

Resumiendo tenemos, que la educación básica, no es un derecho de todos, que no es gratuita, que no conlleva un desarrollo humano pleno, que el acceso a la educación superior no depende de los méritos académicos sino de la situación socioeconómica de las personas y que los padres no tienen libertad de elección respecto al tipo de educación que reciben sus hijos.

Puede afirmarse entonces que la Declaración Universal de los Derechos Humanos en lo referente al ámbito educativo, todavía no se cumple en nuestro país.

\section{Derechos de los niños y educación}

Los Derechos de los Niños, fueron publicados en el año de 1959, el documento enfatiza el derecho a la educación:

En el artículo $7^{\circ}$ afirma:

El niño tiene derecho a recibir educación que será gratuita y obligatoria por lo menos en las etapas elementales. Se le dará una educación que favorezca su cultura general y le permita, en condiciones de igualdad de oportunidades, desarrollar sus aptitudes y su juicio individual, su sentido de responsabilidad moral y social y llegar a ser un miembro útil de la sociedad. El interés superior del niño debe ser el principio rector de quienes tienen la responsabilidad de su educación y orientación; dicha responsabilidad incumbe, en primer término, a sus padres. El niño debe disfrutar plenamente de juegos y recreaciones, los cuales deben estar orientados hacia los fines perseguidos por la educación; la sociedad y las autoridades públicas se esforzarán por promover el goce de este derecho.

En el artículo $2^{\circ}$ expone:

El niño gozará de una protección especial y dispondrá de oportunidades y servicios, dispensado todo ello por la ley y por otros medios, para que pueda desarrollarse física, mental, moral, espiritual y socialmente en forma saludable y normal, así como en condiciones de libertad y dignidad. Al promulgar leyes con este fin, la consideración fundamental 
a que se atenderá será el interés superior del niño.

Se repiten tres elementos que aparecen en la Declaración de los Derechos Humanos: la gratuidad de la educación infantil, su obligatoriedad que correspondería al $100 \%$ de cobertura real y responsabilidad educativa que incumbe prioritariamente a los padres de familia.

Aparece también un aspecto de importancia que nunca se toca en el proyecto educativo mexicano: el niño debe tener oportunidades y servicios para desarrollarse física, mental, moral, espiritual y socialmente en forma saludable.

Ya se ha mencionado el descuido existente en el área física, supondremos que el área mental se desarrolla ya que es el cometido básico de los planes de estudio, el área social seguramente se acrecienta con el simple trato con compañeros; pero la parte moral y sobre todo la espiritual representan temas que no sólo se evaden en la educación mexicana, sino que se observan como peligrosos y obsoletos.

Lo primero que se aprende en educación es que el objetivo, o la intención son básicos para llevarla a buen término, si la "intención educativa nacional" no puede albergar a la persona completa, tampoco puede educarla.

\section{El marco legal de la educación mexicana}

La Constitución de los Estados Unidos Mexicanos da categoría de "garantías individuales" a ciertos derechos que considera indispensables para que el ser humano pueda vivir una vida plena. Una de las primeras condiciones que a este respecto postula es la educación:

Artículo $3^{\circ}$ :

Todo individuo tiene derecho a recibir educación [...] —la cualtenderá a desarrollar armónicamente todas las facultades del ser humano.

Se afirma además que el criterio que orientará dicha educación:

Será democrático, considerando a la democracia [...] como un sistema de vida fundado en el constante mejoramiento económico, social y cultural del pueblo.

Cabe entonces preguntarnos: ¿Es democrática la desigualdad de oportunidades en educación? ¿Qué significa para nuestra Constitución "desarrollar armónicamente todas las facultades"?. ¿Cuáles son esas facultades? ¿Se incluyen en ellas lo moral y lo espiritual? Tal vez la respuesta se encuentra en el segundo párrafo: el criterio orientador debe procurar que el pueblo que se educa adquiera un sistema de vida que le permita mejorar económica, social y culturalmente.

Comparativamente con las declaraciones internacionales se deja ver un panorama incompleto del ser humano, y aún bajo esta poda que nos limita a los elementos económicos, culturales y sociales, siguen existiendo focos de alarma que dan 
cuenta de un patrón que todavía no llega a concretarse a pesar de los innumerables esfuerzos públicos y privados al respecto:

En educación y alimentación el panorama es incierto mostrando un índice de 2.1 millones de niños de 5 a 14 años que no asisten a la escuela, 3.3 millones de niños trabajan y casi uno de cada cinco, menores a cinco años sufre de desnutrición crónica (Cordera, 2005).

Cordera (2005) explica también que existen grupos con mayor vulnerabilidad, como los niños, cuyo desarrollo se ve limitado por la pobreza; especialmente en los estados de Chiapas, Oaxaca, Tabasco, Veracruz, Guerrero e Hidalgo, y las niñas que por el simple hecho de ser niñas no reciben educación.

El problema no se reduce sólo a estos grupos; profundizando en la Ley General de Educación, en su artículo $18^{\circ}$ señala que:

Las instituciones del sistema educativo nacional impartirán educación de manera que permita al educando incorporarse a la sociedad y, en su oportunidad, desarrollar una actividad productiva. (Ley General de Educación. Artº10, VI).

Al observar los índices de desempleo y subempleo de los últimos años, nos cercioramos que la incorporación a la sociedad mediante una actividad productiva no se está cumpliendo. En el caso de los jóvenes, del 60\% entre 15 y 19 años que empezaron a trabajar al mismo tiempo que estudiaban, prácticamente ninguno cuenta con un contrato de trabajo que le permita asumir la vida con criterios de estabilidad. (Encuesta Nacional de la Juventud 2000. Cuadros 104 y 105).

\section{La realidad educativa mexicana vista desde el exterior}

A través de los resultados que arrojan los últimos estudios internacionales, con toda claridad se observa que la mayoría de los niños mexicanos no están siendo preparados adecuadamente para incorporarse a una sociedad globalizada y desarrollar una actividad productiva en ella.

De los informes del estudio PISA de la OCDE (Ischinger 2000) preocupan sobremanera los siguientes datos acerca de la educación básica de nuestro país:

4.1 A pesar de que los fondos destinados a educación aumentaron el $36 \%$ entre 1995 y 2001, la cuota considerada por estudiante ha disminuido dado el aumento de cobertura y la tendencia creciente de la pirámide poblacional en edad escolar. 4.2 Los recursos invertidos en educación resultan insuficientes, y los asignados, son empleados de manera ineficiente; en este renglón, los salarios de los maestros absorben una cantidad desproporcionada del gasto dedicado.

4.3 Existen debilidades en la formación de maestros, en los procedimientos de administración escolar y en las prácticas docentes.

4.4 Aproximadamente el $22 \%$ de los jóvenes de 15 años han reprobado por lo menos un curso de la escolaridad básica; sin embargo, no existen más recursos que la repetición para ayudar a los estudiantes en desventaja. 
4.5 Los resultados obtenidos por nuestros estudiantes son alarmantemente bajos, como ejemplo pondremos el caso de las habilidades matemáticas básicas donde el promedio de resultados de todos los países de la OCDE por debajo del nivel 1 es de $8 \%$, siendo dicho nivel el obtenido por el $38 \%$ de los estudiantes mexicanos. 4.6 Todo parece indicar que los resultados obtenidos en la educación, dependen mucho más de la infraestructura y bagaje cultural de la familia que de la asistencia a la educación escolarizada.

Haciendo cuentas, se observa cómo en nuestro país, ni la educación es una garantía de todos ni es democrática, y a pesar de haberse elevado la cobertura, tampoco es eficiente. Sin embargo, el informe da esperanzas, sugiriendo que los resultados de las evaluaciones se revisen para la toma de decisiones en política educativa; que se dé más peso a las cuestiones pedagógicas que a las administrativas, especialmente en el caso de los directores de escuela y que se promuevan estrategias para atender a los estudiantes con rezago o desventaja de manera que se evite el doble flagelo de la deserción y la reprobación escolar.

\section{La realidad educativa observada desde dentro}

Muchas son las causas que se han sugerido para explicar la falta de eficiencia de la educación mexicana. Dos de ellas, a pesar de ser de orden actitudinal, se hacen evidentes bajo la simple observación de la práctica cotidiana:

En cuanto a los alumnos:

Los estudiantes mexicanos crecen pensando que su educación no les representa un reto, no encuentran estímulos para esforzarse, esto se debe a los siguientes factores:

1) No importa que no aprendas — de cualquier manera pasas al siguiente curso-, esto ocurre en los años más importantes para la formación de hábitos (preescolar, $1^{\circ}$ y $2^{\circ}$ de primaria)

2) Las calificaciones de los estudiantes cumplidos y capaces se diferencian muy poco de las de aquellos que no cubren las metas mínimas, de tal manera que el esfuerzo, se ve constantemente minimizado. Para cerciorarse de esto basta analizar las bases de calificaciones de un grupo escolar de primaria y observar cómo no guardan una relación con la campana de Gauss, prueba clara de que la evaluación se encuentra viciada.

3) La incertidumbre que se genera cuando el alumno es consciente que no sabe lo mismo que sus compañeros, pero que no encuentra forma de resolverlo (el maestro no tiene tiempo para asesorías personales, en la casa no existe la capacidad para ayudarle y no hay estrategias institucionales de recuperación que puedan auxiliarlo). Al cabo de un tiempo, la incertidumbre se convierte en costumbre y el estudiante se asume a sí mismo como incapaz de lograr buenos resultados. Las consecuencias son graves, ya que el educando acarrea dichas carencias desde los niveles más tempranos hasta la educación superior, sin que 
se haga consciente de sus dificultades y responsable de su superación.

En cuanto a los docentes:

A partir de los logros del sindicato que los representa, hay que hacer una doble consideración que lleva a que el docente se perciba a sí mismo como "desechable o sustituible" con la consecuente pérdida de valoración de sí mismo.

Por un lado está la costumbre de heredar (y hasta vender) las plazas, lo cual ha ocasionado, en la práctica, que queden en manos de personas carentes de vocación magisterial; con el afán de encontrar seguridad económica estas personas se incorporan a los cuerpos docentes devaluando el carisma de la profesión.

Por otro lado, los innumerables permisos, comisiones y becas a que puede hacerse acreedor todo maestro, que ciertamente son necesarios y que le sirven para maximizar sus esfuerzos en la práctica docente, también en muchas ocasiones sirven para albergar la dejadez y la doble moral, además de representar una enorme carga económica para el país.

Poniendo el panorama de la peor manera posible, si un maestro escogió su profesión por considerarla un servicio genuino y fundamental para el desarrollo del país, y durante su trayectoria profesional observa continuamente a otros que, evitando el trabajo, obtienen lo mismo, heredan sus plazas a descendientes incapaces y ocasionan además que la profesión se devalúe ante la mirada social, lo más fácil es caer en la desesperación y dejar de valorarse a sí mismo.

Resumiendo: Tenemos una normatividad que considera un ser humano incompleto; alumnos que no saben por qué, ni cómo esforzarse; docentes que en buena medida han perdido la noción de su importante labor y una población que ha perdido la fe en la educación ya que sus malos resultados han sido pregonados por todos los medios.

La única carta que puede proveer al país de un futuro esperanzador, es el cambio del paradigma educativo y ese cambio sólo puede darse desde los propios agentes de la educación, los docentes.

\section{La dignificación del maestro al rescate de la educación}

La importancia del maestro para romper el círculo vicioso en que se encuentra la educación es indiscutible, el maestro es capaz de hacer que los estudiantes conciban la vida educativa como un reto alcanzable a través del propio esfuerzo; el maestro es capaz de multiplicar conceptos, ideas, valores, y con ello alterar el desarrollo de la vida futura de una nación, de ahí que resulte crucial su dignificación.

La idea de "dignificar al magisterio" no es un concepto de interés nacional, sino mundial. ${ }^{1}$

En México nos referimos a la dignificación del maestro englobando dos aspectos fundamentales: sus condiciones salariales y la actualización necesaria para 
mejorar técnica y pedagógicamente su labor.

La pregunta que debemos hacernos es si el maestro mexicano, solamente necesita elevar su salario y prepararse mejor para dignificarse, esto es, para sentirse un ser humano digno y que la sociedad le guarde el respeto debido en consideración a dicha dignidad.

Antonio Botana (2006) refiere tres niveles de identidad del docente, los cuales conviene considerar porque aclaran las probabilidades de éxito de los proyectos de dignificación magisterial. Los tres niveles son necesarios para el educador, pero cada uno debe elegir aquel que le resulte más importante como elemento motivador de la acción cotidiana:

7.1 El primer nivel corresponde al plano biológico-laboral, en él, el maestro se asume como un trabajador de la enseñanza y debe satisfacer las necesidades de subsistencia básicas; la motivación es la supervivencia y se consigue mediante un salario justo.

7.2. El segundo nivel está en el plano psicológico-social en el que el maestro se asume como un profesional de la enseñanza con dominio de las asignaturas y las metodologías adecuadas para enseñarlas. La motivación consiste en sentirse socialmente valorado y se consigue a partir de procesos de educación continua.

7.3. El tercer nivel llega al núcleo de la identidad, al "ser" de la persona y el maestro se asume no sólo como trabajador o profesional de la enseñanza, sino como auténtico educador que "tiene la impresión de estar ocupando el lugar adecuado en la sinfonía de la creación" (Botana p.9). La motivación es el servicio y se cubre en la confluencia de la supervivencia, la autovaloración y la vocación.

Para entenderlo con mayor claridad, Botana se refiere al educador "vocacionado" lo que comúnmente llamamos un maestro con vocación.

Al revisar estos niveles, no podemos dejar de pensar en la multitud de maestros mexicanos "vocacionados", que se pierden en el laberinto de las discusiones salariales y en los esquemas de profesionalización con puntajes para escalafón.

$\mathrm{Ni}$ los esfuerzos actuales de dignificación magisterial, ni la Constitución, ni la Ley General de Educación se refieren a este tercer nivel que es fundamental para que el maestro mexicano reencuentre la dignidad, porque la dignidad no puede venir de fuera, tiene que percibirse desde el interior del ser humano, desde su propia identidad. El tercer nivel supone aceptar el reto de sentirse indispensable para los educandos que se tienen delante cada día; sin el maestro enfrente, su vida debería ser radicalmente diferente.

Lo anterior no quiere decir que el maestro debe conformarse con sueldos miserables o dejar de prepararse, los tres niveles importan; pero cuando las cosas se observan desde la profundidad de la vocación, suelen tomar una dimensión diferente.

Para concluir este inciso baste decir que el educador de tercer nivel no sólo siente 
la fuerza de su identidad sino que influye en la comunidad educadora y en la organización escolar. Se sabe digno y la comunidad reconoce su dignidad, ésta es la verdadera dignificación magisterial. La gran diferencia es que independientemente de la motivación por los intereses laborales o el prestigio académico, el docente "vocacionado" se empeña por poner en el centro de su labor a sus alumnos y su intención se dirige a cubrir las necesidades de cada uno de la mejor manera posible.

\section{Propuesta de dignificación del magisterio}

Juan Bautista de La Salle fue el primero en preocuparse por la formación de maestros, ya en el siglo XVII (Gallego p.39) se daba cuenta que el buen maestro era la única posibilidad de tener éxito en la educación.

A partir de la experiencia resuelve la necesidad de acompañamiento del maestro formando comunidades en las que de manera fraternal pudieran tratarse asuntos de índole académica y personal, tanto de los alumnos como de sí mismos.

Los logros de La Salle fueron tan importantes en la Francia de su época que se consideraba al maestro lasallista como el ideal docente, a pesar de que tratarse en muchos casos de hombres burdos y con una preparación académica mínima.

Según el informe de la OCDE, los maestros mexicanos de hoy tienen carencias en su formación; pero tienen muchas más competencias que los maestros lasallistas del tiempo citado; también cuentan con gran cantidad de cursos de actualización y con un sindicato que vela por la cobertura de su necesidades básicas.

Volviendo entonces al esquema de Botana, podemos decir que los docentes mexicanos pueden encontrar la forma de cubrir los primeros dos niveles de dignificación, pero en muchos casos carecen de la posibilidad de llegar al tercer nivel dado que la legislación mexicana no considera los aspectos morales ni espirituales y se limita a presentar la educación como un modelo laico.

La hipótesis que se propone es la siguiente: "Si los docentes mexicanos pueden acceder a un espíritu que los albergue y los lleve a desarrollarse en comunidad, la educación mexicana superará sus principales carencias de manera natural y sin mayor gasto de recursos".

\section{CONCLUSIÓN}

México como nación ha firmado y estado de acuerdo tanto con la Declaración Universal de los Derechos Humanos como con la Declaración de los Derechos de los Niños, en ambos se considera una parte espiritual dentro del ser humano que debe incluirse en los proyectos de desarrollo y educación.

La normatividad mexicana, sin embargo, pasa por encima de este rubro sin siquiera exponerlo, como si no existiera.

Quedan entonces dos caminos: o como nación aceptar que no estamos de acuerdo en que existe una parte espiritual en el ser humano, o admitir que existe y empezar a hacernos cargo de ella. 
Se entiende que esta situación procede de episodios históricos en que el protagonismo de las instancias religiosas resultaba peligroso para el desarrollo nacional. Pero pasa el tiempo y en un país que sigue siendo mayoritariamente religioso, es decir, que cree en una parte espiritual, se sigue queriendo tapar el sol con un dedo.

No se trata de querer volver al pasado, sino de asumir que la vocación magisterial tiene un fuerte contenido espiritual que no puede ser paliado con cursos o sueldos, sino que requiere alimentarse de elementos de su mismo orden y que en la medida en que el docente se encuentre nutrido, podrá a su vez trasladar esas actitudes a los educandos que cotidianamente forma.

En la medida en que esto empiece a considerarse, el maestro mexicano podrá rendir no sólo lo que su salario o su preparación profesional le permitan, sino lo que su completa identidad le impulse a dar, esta es quizás la única forma de alterar el devenir de la educación mexicana.

\section{NOTAS}

${ }^{1}$ Pueden consultarse las siguientes páginas para analizar los casos de Perú, Nicaragua y la Comunidad de Murcia en España respectivamente www.tarea.org.pe/modulos/boletin/tareainforma; http://www.oei.es/linea6/nicaragua.htm; www.rural.carm.es/.../archivoMultimedia

\section{FUENTES DE CONSULTA}

Botana, Antonio F. (2006) Cuadernos MEL 8y 9. Itinerario del educador. www.lasalle.org

(recuperado el 11 de diciembre de 2006)

Constitución Política de los Estados Unidos Mexicanos

Cordera Campos, Rolando (2005) en

http://www.esmas.com/noticierostelevisa/investigaciones /479859.html oct. 4, 2005. (Recuperado el 12 de febrero de 2007)

Encuesta Nacional de la Juventud. 2000. SEP. Instituto Mexicano de la juventud. Centro de Investigación y estudios sobre juventud.

Declaración de los Derechos del Niño (1959), aprobada por la Asamblea General de las Naciones Unidas el 20 de noviembre de 1959.

Declaración Universal de los Derechos humanos.

http://www.un.org/spanish/aboutun/hrights.htm (Recuperado el 5 de marzo de 2007)

Ley General de Educación. Publicada en el Diario oficial de la Federación el 13 de julio de 1993. Última reforma: 13 de marzo de 2003.

http://www.margen.org/ninos/derech4a.html (Recuperado el 28 de febrero de 2007)

Gallego, Ma. Cristina. (s/a) De La Salle. Un hombre para la eternidad. Universidad La Salle Pachuca 
ISCHINGER, Barbara. (2000) Informe OCDE.

http://www.foropoliticaspublicas.org.mx/docs/Educación Barbara\%20lschinger 0 CDE.pdf (Recuperado el 5 de marzo de 2007) 